\title{
La calidad del servicio y su influencia en la satisfacción de los estudiantes de la facultad de ciencias jurídicas y empresariales de la universidad nacional Jorge Basadre Grohmann de Tacna, 2012- 2013.
}

Quality of service and its influence on students' satisfaction of the faculty of business and judicial sciences of Jorge Basadre Grohmann national university of Tacna, 2012-2013.

Jerzy Alférez Asencios ${ }^{1}$

\section{RESUMEN}

Objetivo: El presente trabajo de investigación se llevó a cabo con el propósito de determinar la influencia de la calidad de servicio en la satisfacción de los estudiantes de la Facultad de Ciencias Jurídicas y Empresariales de la Universidad Nacional Jorge Basadre Grohmann de Tacna.

Método: El trabajo corresponde como una investigación de tipo básica y nivel correlacional. Para tal propósito se consideró la información obtenida a través de la aplicación del instrumento de medición del cuestionario para medir la calidad de servicio y satisfacción que se brinda en la Facultad de Ciencias Jurídicas y Empresariales, los cuales fueron aplicados a los estudiantes utilizándose la escala de Likert. Finalizada la fase de análisis e interpretación de los resultados se determinó que en la Facultad de Ciencias Jurídicas y Empresariales, la calidad de servicio influye en la satisfacción de sus estudiantes. El modelo de medición consta de 10 indicadores de calidad de servicio y 22 indicadores de satisfacción de estudiantes.

Resultado: Se han determinado que la calidad de servicio es percibida como negativa por un $49.97 \%, 16.71$ es percibida como positiva y un $33.32 \%$ es indiferente. Por otro lado, La Satisfacción de estudiantes, $30.47 \%$ se encuentra satisfecho, $33.15 \%$ se encuentra insatisfecho y un $36.38 \%$ se encuentra indiferente.

Conclusiones: Las dimensiones de Calidad de servicio como elementos tangibles, confiabilidad, capacidad de respuesta, seguridad y empatía son percibidas como calidad pésima, y las dimensiones de satisfacción como métodos de enseñanza y aprendizaje, organización de las carreras e infraestructura de la facultad indican insatisfacción, mientras que las dimensiones evaluaciones y equipo docente indican indiferencia de los estudiantes.

\begin{abstract}
Objective: The present research was conducted in order to determine the influence of quality of service on students' satisfaction of The Faculty of Business and Judicial Sciences of Jorge Basadre Grohmann National University of Tacna.
\end{abstract}

Method: The work corresponds as a basic research type and correlational level. For this purpose it was considered information obtained through the application of the questionnaire measuring instrument for assessing the quality of service and satisfaction provided in The Faculty of Business and Judicial Sciences, which were applied to students with technical survey, using the Likert scale. After the phase of analysis and interpretation of the results was determined that in the Faculty Business and Judicial Sciences, quality of service influences on students' satisfaction. The measurement model has 10 indicators of quality of service and 22 indicators for students' satisfaction.

Result: They have determined that quality of service is perceived as negative by $49.97 \%$, $16.71 \%$ is perceived as positive and $33.32 \%$ is indifferent. In other hand, students satisfaction, $30.47 \%$ are satisfied, $33.15 \%$ are insatisfied and $36.38 \%$ are indiferent.

Conclusions: Quality of service dimensions as tangible elements, fiability, answer capability, security and empathy are perceived as poor quality, and satisfaction dimensions as learning and teaching methods, organization and infrastructure career Faculty indicate insatisfaction, while the dimensions and teaching equipment evaluations indicate indifference of Students

\section{KEYWORDS}

Quality of service, satisfaction, students, survey, poor quality. 


\section{Introducción}

La calidad de servicio está tomando mucho auge debido a que es indispensable en todo tipo de organización que busca mejorar sus servicios y generar satisfacción en sus clientes o usuarios.

En esta investigación se busca tener una mejor comprensión acerca de la importancia de la calidad de servicio y satisfacción del estudiante.

La calidad no es un tema nuevo ya que desde los tiempos de los jefes tribales, reyes y faraones han existido los argumentos y parámetros sobre calidad. El Código de Hammurabi (1752 a. C.), declaraba: "Si un albañil construye una casa para un hombre, y su trabajo no es fuerte y la casa se derrumba matando a su dueño, el albañil será condenado a muerte". Los inspectores fenicios, cortaban la mano a quien hacía un producto defectuoso, aceptaban o rechazaban los productos y ponían en vigor las especificaciones gubernamentales. Alrededor del año 1450 a. C., los inspectores egipcios comprobaban las medidas de los bloques de piedra con un pedazo de cordel. Los mayas también usaron este método. La mayoría de las civilizaciones antiguas daban gran importancia a la equidad en los negocios y cómo resolver las quejas, aun cuando esto implicara condenar al responsable a la muerte, la tortura o la mutilación. (Wikipedia).

Con la ayuda de la Revolución industrial, surgida en Gran Bretaña con la aparición de la máquina de vapor, la producción en masa de productos manufacturados se hizo posible mediante la división del trabajo propuesta por Adam Smith en su obra La riqueza de las naciones y la creación de partes intercambiables; sin embargo, esto creó problemas para los que estaban acostumbrados a que sus productos fueran hechos a la medida.

En 1950 W. Edwards Deming, un hombre dedicado a la estadística que había trabajado en la Bell System con George Edwards y Walter A.

Shewhart, fue invitado a hablar ante los principales hombres de negocios del Japón, quienes estaban interesados en la reconstrucción de su país al término de la Segunda Guerra Mundial, e intentando entrar en los mercados extranjeros y cambiando la reputación del Japón de producir artículos de calidad inferior. Deming los convenció de que la calidad japonesa podría convertirse en la mejor del mundo al instituirse los métodos que él proponía.

Muchas empresas comienzan a trabajar con el concepto de "Sistema Integral de Calidad", que afecta al diseño, la fabricación y la comercialización, produciéndose un fenómeno singular que afectó a la comercialización y economía industrial de muchos países, como consecuencia del despegue de la industria japonesa, aplicando los conceptos del aseguramiento de la calidad y la prevención.

Los industriales japoneses aprendieron las enseñanzas de Deming y la calidad japonesa, la productividad y su posición competitiva se mejoraron y reforzaron, para ser lo que son hoy en día. Es por ello que cada año se otorga en el Japón los muy deseados Premios Deming al individuo que muestre logros excelentes en teoría o en la aplicación del control de la calidad por estadísticas, o aquella persona que contribuya notablemente a la difusión de las técnicas del control de calidad por estadísticas, así como a su aplicación. Las compañías japonesas que han obtenido dichos premios incluyen Nissan, Toyota, Hitachi y Nipon Steel. En 1989, la Florida Power and Light Company fue la primera 
compañía extranjera en ganar el premio Deming.

El concepto de calidad proviene del ámbito económico industrial tomando mayor impulso a medida que la economía se tornó más competitiva convirtiéndose gradualmente en una variable cada vez más importante en la elaboración y prestación de bienes y servicios. Todo este proceso aceleró la adopción de distintas estrategias organizacionales que van desde la tecnificación empresarial hasta la capacitación de los recursos humanos.

En el sistema educativo también se busca la calidad. La acreditación de la calidad y el seguimiento de criterios homogéneos en su evaluación son dos de los retos a los que se enfrentan las universidades peruanas. La acreditación de la calidad educativa se basa en la Ley 28740, promulgada el 19 de mayo del dos mil seis, Ley del Sistema Nacional de Evaluación, Acreditación y Certificación de la Calidad Educativa (SINEACE) cuyo Reglamento que ha sido publicado el 10 de julio del dos mil siete (Universia).

El Congreso aprobó la "Ley que Establece la Moratoria para la Creación de Universidades Públicas y Privadas por un período de Cinco Años". La ley "impide la creación y autorización de funcionamiento de nuevas universidades públicas y privadas" y "suspende la creación de filiales y el ingreso en la modalidad de educación a distancia". También autoriza "excepcionalmente, por el plazo de un año, al Consejo Nacional para la Autorización de Funcionamiento de Universidades (CONAFU), para que evalúe y autorice a las universidades que cuenten con el Proyecto de Desarrollo Institucional (PDI) aprobado para la fase de implementación" (Instituto Peruano de Economía, 2012).
En la actualidad, se ha acentuado la exigencia respecto a la competitividad del profesional en el campo laboral, su éxito dependerá en gran medida de la calidad de la educación obtenida y de la formación práctica para el trabajo; es muy importante que la facultad se encuentre inmersa en un proceso de permanente mejoramiento con una visión orientada hacia el futuro y en beneficio del estudiante.

En la Facultad de Ciencias Jurídicas y Empresariales de la Universidad Nacional Jorge Basadre Grohmann de Tacna, la calidad que perciben los estudiantes es negativa debido a los siguientes aspectos:

Existen demoras en la atención a las necesidades de los estudiantes de la Facultad debido al tiempo que tienen que esperar en sus trámites administrativos.

No se percibe una buena relación de amistad y confianza entre profesor y alumno, ni administrativo y alumno.

Las infraestructuras de las escuelas profesionales no son modernas, así como también no se encuentran implementados todos los salones de clases con equipos multimedia.

Los estudiantes de la Escuela Profesional de Contabilidad están estudiando en diferentes escuelas y diferentes horarios debido a que la infraestructura de su edificio está siendo refaccionada.

La percepción de buena calidad por parte de los alumnos hace que se sientan inclinados a recomendar la institución a otros y volver a los alumnos leales que usarán todas las oportunidades para apoyar a sus escuelas, por lo tanto, el propósito de esta investigación busca demostrar el valor de la calidad de servicio en la 
Facultad de Ciencias Jurídicas y Empresariales.

Para Deming la calidad debe tener como objetivo las necesidades del usuario, presentes y futuras. Para Juran representa la adecuación a la finalidad 0 al uso. Para Crosby, es la conformidad con las exigencias. Feigenbaum dice que es lo total de las características de un producto $\mathrm{o}$ servicio referente a marketing, ingeniería, manufactura y mantenimiento, por las cuales el producto o servicio, cuando en uso, atenderá las expectativas del cliente (2006).

Para Rosander, A. (1994), servicio es una situación cara a cara en la que el cliente trata directamente con el vendedor. Calidad de servicio es lo que el cliente recibe de estas personas. La calidad de servicio no viene del Director General. A menos que la alta dirección entienda que la mejora de la calidad es un programa continuo y convenza a todos los empleados para adoptar el mismo punto de vista, esos vendedores no darán calidad de servicio.

Calidad de servicio se asocia igualmente con trabajadores profesionales, quienes determinan la calidad de servicio que el cliente va a recibir. Esos trabajadores van desde médicos a mecánicos de automóviles.

Un médico diagnostica una enfermedad. Calidad de servicio significa un diagnóstico correcto y un tratamiento eficaz para curar al paciente. Un mecánico de automóviles debe realizar también un diagnóstico correcto y eliminar la causa del problema. Esto es el cumplimiento de la calidad: eliminar problemas.

La satisfacción de los alumnos equivale a lo que en las empresas se denomina satisfacción de los clientes externos, aunque también son clientes internos en la medida en que son coagentes de su educación. Ésta ha de basarse en la atención a sus necesidades $y$ posibilidad educativas y al logro de las expectativas que se le planteen (Rodríguez C.).

Según Vavra (2002) la Satisfacción de Clientes es un estado emocional positivo del cliente ante un servicio o producto después de haberlo experimentado.

Para Castillo C. (2002), la satisfacción de los alumnos guarda cierta similitud con lo que en una empresa de productos o servicios se define como "satisfacción de los clientes externos", por cuanto se refiere a los destinatarios inmediatos a quienes se ofrece el producto educativo. Cierto que en una institución educativa los alumnos son, además, co-agentes en la determinación del producto educativo (lo que los sitúa dentro del personal del centro, con el que formarían los cliente internos); pero, siquiera sea por razones de metodología de estudio, consideramos aquí este predictor desde la perspectiva de su condición de receptores del producto que se elabora en un centro o institución educativa.

\section{Materiales y métodos}

Es una investigación básica, porque se describe un problema en una realidad. De acuerdo a la naturaleza del estudio de la investigación, reúne las características de un estudio correlacional porque busca una relación entre la variable independiente y variable dependiente.

La investigación se realizó en la Facultad de Ciencias Jurídicas y Empresariales de la Universidad Nacional Jorge Basadre Grohmann de Tacna en el año académico 2013 y la 
muestra estuvo conformada por 301 estudiantes.

En dicha Facultad se dictan las carreras profesionales de Administración, Ciencias Contables y Financieras, Derecho y Ciencias Políticas e Ingeniería Comercial.

Para la recolección de datos se empleó el instrumento de medición del cuestionario aplicado bajo la técnica de la encuesta basado en la Calidad de Servicio y la Satisfacción de los Estudiantes a la cual se le aplicó el índice de Cronbach para ser confiable. Dicho instrumento de medición fue distribuido a los estudiantes de la Facultad de Ciencias Jurídicas y Empresariales, los días 25 de noviembre al 06 de diciembre del 2013.

\section{Resultados}

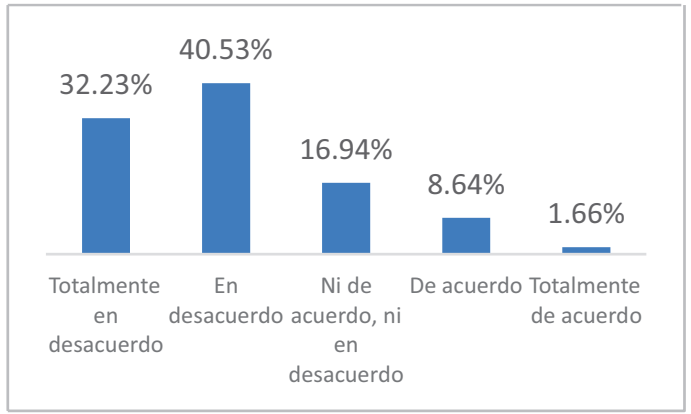

Figura 1

La Facultad cuenta con máquinas y equipos de apariencia moderna y atractiva

Del análisis se concluye que un $10.30 \%$ de los encuestados mantiene una posición De acuerdo y Totalmente de acuerdo, mientras que un $16.94 \%$ mantiene una posición $\mathrm{Ni}$ de acuerdo, ni en desacuerdo, y un $72.76 \%$ mantiene una posición En desacuerdo y Totalmente en desacuerdo, porque no observa computadoras, equipos multimedia ni impresoras de última generación en su facultad.

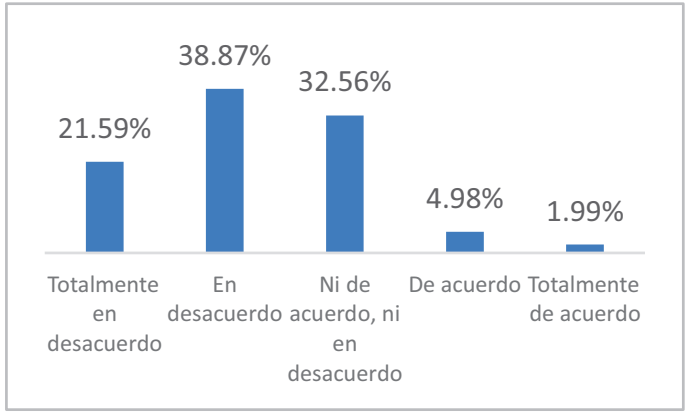

Figura 2

Cuando tienes un problema, la Facultad muestra un sincero interés en solucionártelo

Del análisis se concluye que un $6.98 \%$ de los encuestados mantiene una posición De acuerdo y Totalmente de acuerdo porque los administrativos ayudaron en algún trámite de manera correcta, mientras que un $32.56 \%$ mantiene una posición $\mathrm{Ni}$ de acuerdo, ni en desacuerdo, y un $60.47 \%$ mantiene una posición En desacuerdo y Totalmente en Desacuerdo porque los administrativos de la Facultad no atendieron sus problemas.

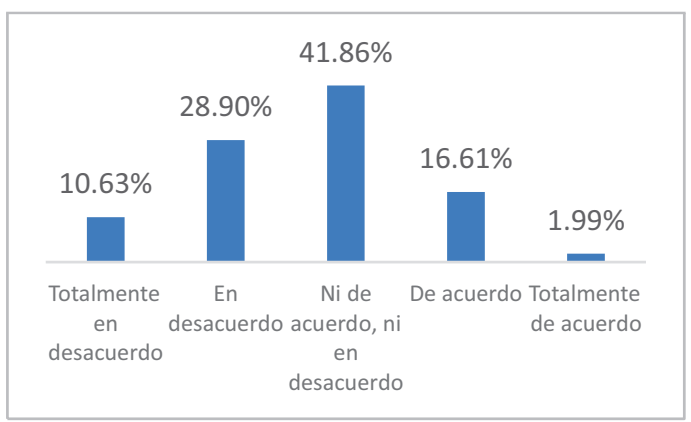

Figura 3

El personal de la Facultad siempre está dispuesto a ayudarle

Del análisis se concluye que un $18.60 \%$ mantiene una posición De acuerdo y Totalmente de acuerdo, porque los administrativos han ayudado a los alumnos, mientras que un $41.86 \%$ mantiene una posición $\mathrm{Ni}$ de acuerdo, ni en desacuerdo, y un $39.53 \%$ mantiene una posición En desacuerdo y Totalmente en desacuerdo, porque los administrativos ayudan a los alumnos cuando tienen problemas. 


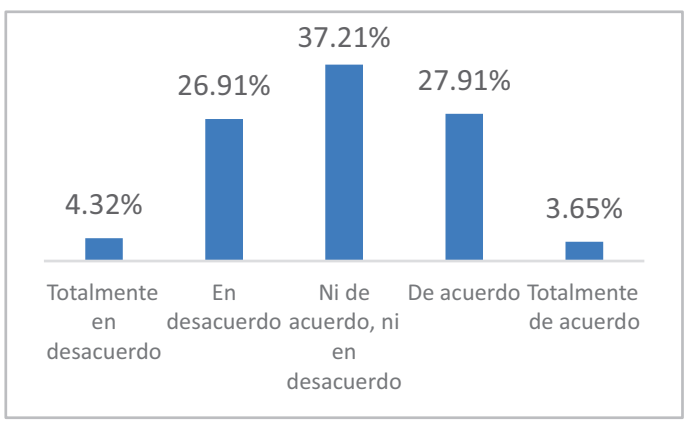

Figura 4

El personal tiene conocimientos suficientes para responder a sus preguntas

Del análisis se concluye que un $31.56 \%$ de los encuestados mantiene una posición De acuerdo y Totalmente de acuerdo, porque el personal resuelve sus dudas, mientras que un $37.21 \%$ mantiene una posición $\mathrm{Ni}$ de acuerdo, ni en desacuerdo, y un $31.56 \%$ mantiene una posición mantiene una posición En desacuerdo y Totalmente en desacuerdo, porque el personal no resuelve sus dudas.

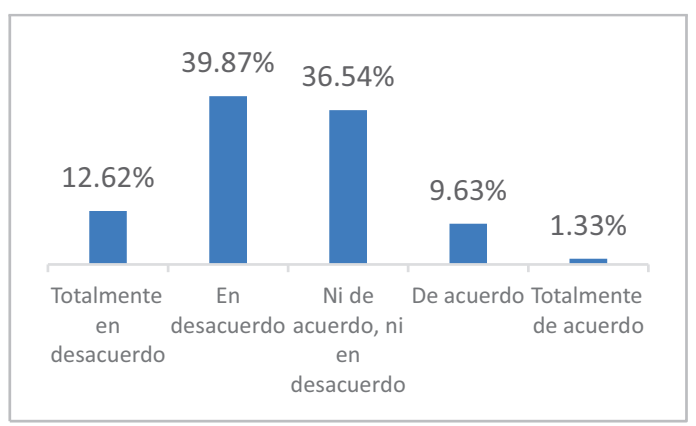

Figura 5

La Facultad comprende las necesidades específicas de sus estudiantes

Del análisis se concluye que un $10.96 \%$ de los encuestados mantiene una posición De acuerdo y Totalmente de acuerdo, porque los administrativos entienden los pedidos de los alumnos, un $36.54 \%$ mantiene una posición $\mathrm{Ni}$ de acuerdo, ni en desacuerdo, y un $52.49 \%$ mantiene una posición En desacuerdo y Totalmente en desacuerdo, porque los administrativos no entiende los pedidos de los alumnos.

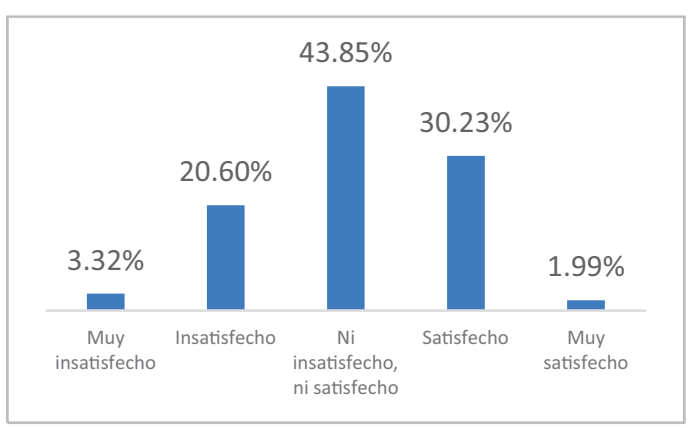

Figura 6

El docente motiva a participar en clases

Del análisis se concluye que un $32.23 \%$ se encuentra Satisfecho y Muy satisfecho porque el docente aplica técnicas para motivar a sus alumnos y participen en clases, mientras que un $43.85 \%$ mantiene una posición ni insatisfecho, ni satisfecho, y un $23.92 \%$ se encuentra insatisfecho y muy insatisfecho porque el docente no motiva a que los estudiantes para que participen en clases sino por obligación para no ser desaprobados.

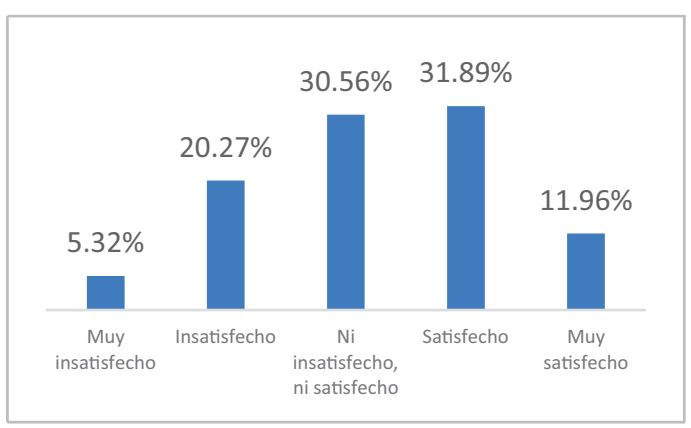

Figura 7

Las clases prácticas son útiles

Del análisis se concluye que un $43.85 \%$ de los encuestados se encuentra Satisfecho y Muy satisfecho, porque las clases prácticas les permiten aprenden más y aplicar sus conocimientos teóricos, mientras que un $30.56 \%$ mantiene una posición $\mathrm{Ni}$ insatisfecho, ni satisfecho, y un $25.58 \%$ se encuentra Insatisfecho y Muy insatisfecho porque no encuentra interés en las clases prácticas. 


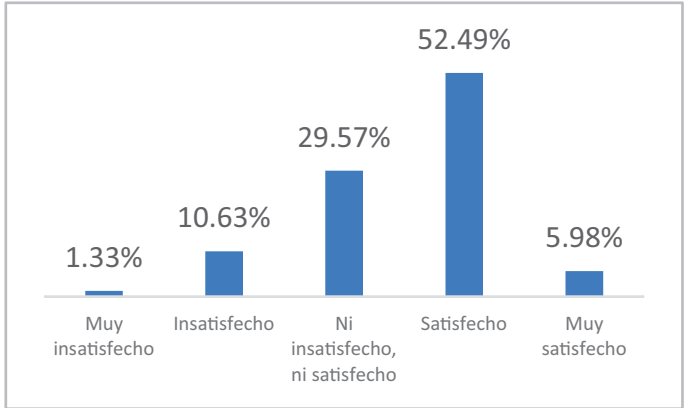

Figura 8

Se avisa oportunamente sobre las fechas de trabajos prácticos

Del análisis se concluye que un $58.47 \%$ de los encuestados se encuentra Satisfecho y Muy satisfecho, porque el docente les avisa con tiempo la fecha para la presentación de trabajos prácticos y sustentarlos debidamente, mientras que un $29.57 \%$ mantiene una posición Ni insatisfecho, ni satisfecho, y un $11.96 \%$ se encuentra Insatisfecho y Muy insatisfecho porque el docente no avisa con tiempo las fechas de trabajos prácticos y deja poco tiempo para prepararse para la exposición.

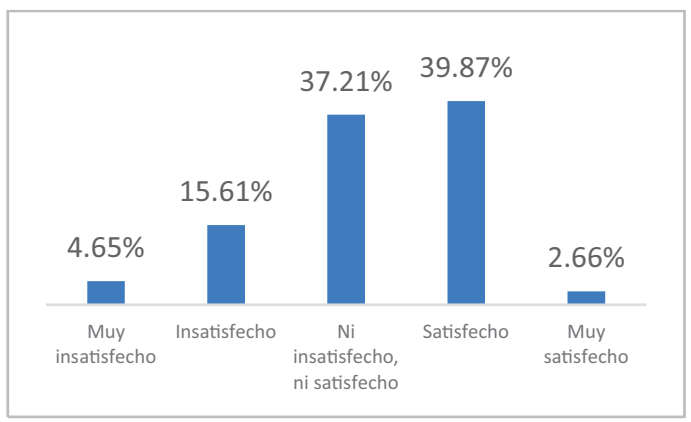

Figura 9

Existe coherencia con la materia enseñada en clases

Del análisis se concluye que un $42.52 \%$ se encuentra Satisfecho y Muy satisfecho porque las preguntas de los exámenes si tienen relación con la materia enseñada, mientras que un $37.21 \%$ mantiene una posición $\mathrm{Ni}$ insatisfecho, ni satisfecho, y un $20.27 \%$ se encuentra Insatisfecho y Muy insatisfecho porque las pruebas son más complicadas que lo enseñado en clases $o$ no se entiende el tema redactado, pudiendo preguntar al docente las dudas para desarrollar el examen.

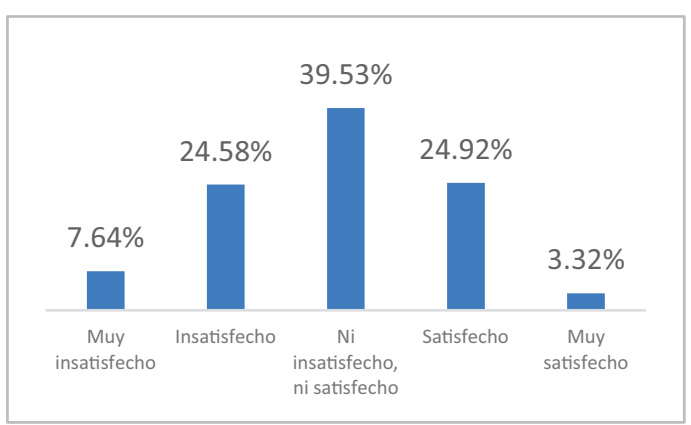

Figura 10

Disposición para analizar y discutir las evaluaciones

Del análisis se concluye que un $28.24 \%$ de los encuestados se encuentra Satisfecho y Muy satisfecho porque se realiza la corrección de los exámenes, mientras que un $39.53 \%$ mantiene una posición $\mathrm{Ni}$ insatisfecho, ni satisfecho, y un $32.23 \%$ se encuentra Insatisfecho y Muy insatisfecho porque no se corrigen los exámenes.

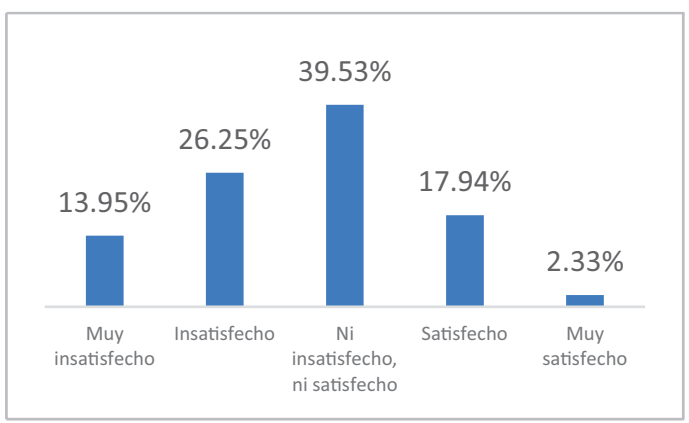

Figura 11

Existe calidad de atención en Secretaría

Del análisis se concluye que un $20.27 \%$ de los encuestados se encuentra Satisfecho y Muy satisfecho porque en Secretaria los atienden bien cuando presentan alguna solicitud, mientras que un $39.57 \%$ mantiene una posición $\mathrm{Ni}$ insatisfecho, ni satisfecho, y un $40.20 \%$ se encuentra Insatisfecho y Muy insatisfecho porque en Secretaría nos los atienden de buena manera. 


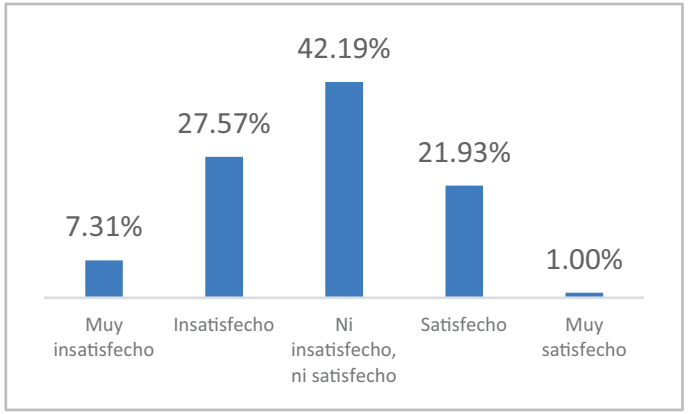

Figura 12

Existe coherencia entre las asignaturas cubiertas por la Malla Curricular

Del análisis se concluye que un $22.92 \%$ de los encuestados se encuentra Satisfecho y Muy satisfecho porque los cursos enseñados son útiles en su campo laboral, mientras que un $42.19 \%$ mantiene una posición $\mathrm{Ni}$ insatisfecho, ni satisfecho, y un $34.88 \%$ se encuentra Insatisfecho y Muy insatisfecho porque los cursos no tienen coherencia con su campo laboral.

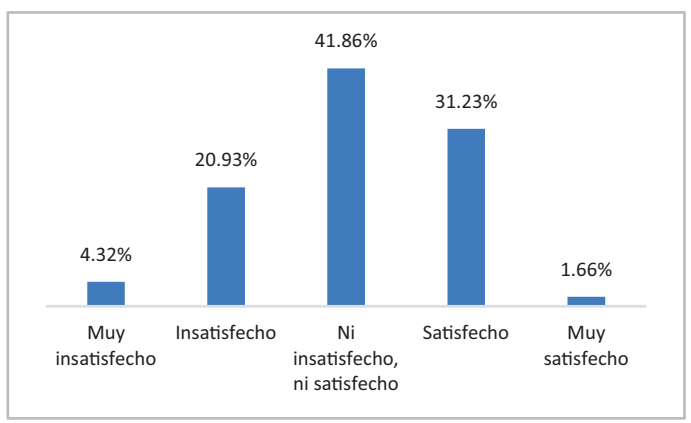

Figura 13

Existe calidad académica del profesorado

Del análisis se concluye que un $32.89 \%$ de los encuestados se encuentra Satisfecho y Muy satisfecho porque casi todos los docentes tienen grados de magister y experiencia profesional y metodología de enseñanza, mientras que un $41.86 \%$ mantiene una posición $\mathrm{Ni}$ insatisfecho, ni satisfecho, y un $25.25 \%$ se encuentra Insatisfecho y Muy insatisfecho porque algunos profesores no tienen grados de magister ni metodología para enseñar.

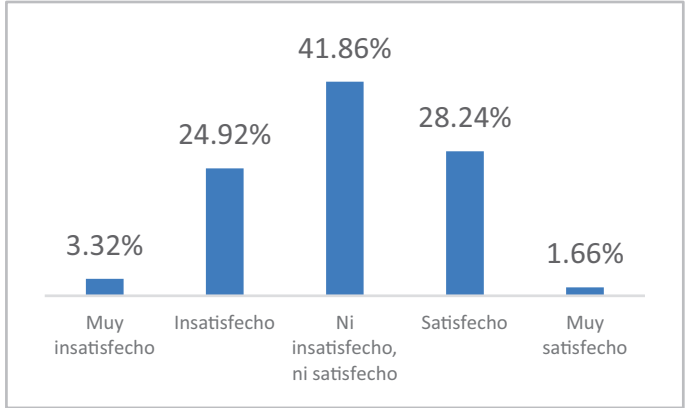

Figura 14

Puntualidad y cumplimiento de horas de clases

Del análisis se concluye que un $29.90 \%$ de los encuestados se encuentran Satisfecho y Muy satisfecho porque los docentes comienzan sus clases puntuales y terminan respetando los tiempos, mientras que un $41.86 \%$ mantiene una posición $\mathrm{Ni}$ insatisfecho, ni satisfecho, y un $28.24 \%$ se encuentra Insatisfecho y Muy insatisfecho porque los profesores llegan tarde y terminan sus clases antes de tiempo.

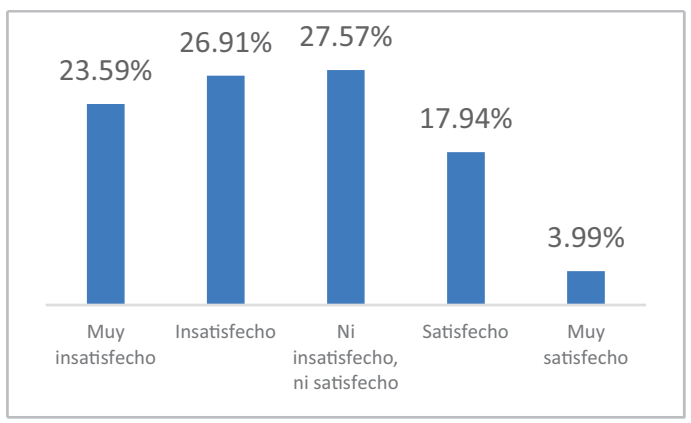

Figura 15

Existe disponibilidad del laboratorio de cómputo

Del análisis se concluye que un $21.93 \%$ de los encuestados se encuentra Satisfecho y Muy satisfecho porque tienen tiempo para usar el laboratorio de cómputo previa coordinación con el encargado del laboratorio, mientras que un $27.57 \%$ mantiene una posición $\mathrm{Ni}$ insatisfecho, ni satisfecho, y un $50.50 \%$ se encuentra Insatisfecho y Muy insatisfecho porque no tiene de usar el laboratorio de cómputo debido al 
tiempo o a un cruce de horarios con otras secciones.

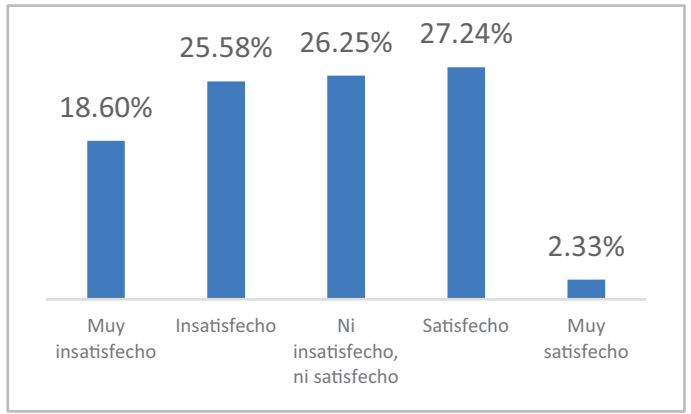

Figura 16

Existe disponibilidad de libros y material bibliográfico en la biblioteca

Del análisis se concluye que un $29.57 \%$ de los encuestados se encuentra Satisfecho y Muy satisfecho porque existe material bibliográfico disponible en la biblioteca para realizar sus tareas y trabajos prácticos, mientras que un $26.25 \%$ mantiene una posición $\mathrm{Ni}$ insatisfecho, ni satisfecho, y un $44.19 \%$ se encuentra Insatisfecho y Muy insatisfecho porque no encuentra material útil en la biblioteca, y debe revisar más material en otras bibliotecas.

\section{Discusión}

En relación a la variable Calidad de Servicio, en la Dimensión de Elementos tangibles se obtuvo una media de 2.28 , debido a que los estudiantes perciben que las máquinas y equipos no son modernos así como las instalaciones físicas no son visualmente atractivas.

En la Dimensión Confiabilidad se obtuvo 2.42, debido a que los estudiantes perciben que cuando tiene un problema la Facultad no toma interés en solucionarlo y el personal no realiza bien sus servicios en el tiempo prometido.

En la Dimensión Capacidad de Respuesta se obtuvo una media de 2.63, debido a que los estudiantes perciben que el personal no ofrece un servicio puntual, así como tampoco están siempre dispuestos a ayudarlos.

En la Dimensión Seguridad se obtuvo una media de 2.92, debido a que los estudiantes perciben que el personal no es siempre amable con ellos y tienen conocimientos regulares para responder a sus preguntas.

En la Dimensión Empatía se obtuvo una media de 2.52, debido a que los estudiantes perciben que el personal no les ofrece una atención personalizada.

Estos resultados indican una mala calidad percibida por los estudiantes siendo las puntuaciones más deficientes.

En relación a la variable Satisfacción del Estudiante, en la Dimensión Métodos de Enseñanza y Aprendizaje se obtuvo una media de 2.88 , debido a que los estudiantes son indiferentes en relación a las clases teóricas si el docente los motiva a participar en clases y la entrega de material útil, así como la utilidad de las clases prácticas; y también se encuentran insatisfechos en relación a que no es adecuada la cantidad de clases prácticas y el apoyo que brindan para realizar prácticas preprofesionales.

En la Dimensión Evaluaciones se obtuvo una media de 3.19 , debido a que los estudiantes son indiferentes si se avisan oportunamente las fechas de evaluaciones y trabajos prácticos, si existe coherencia entre la materia enseñada con las evaluaciones y la redacción de las evaluaciones; y también se encuentran insatisfechos sobre la disposición para analizar y discutir las evaluaciones y procedimientos de apelación respecto de notas dadas.

En la Dimensión Organización de las Carreras se obtuvo una media de 2.71, 
debido a que los estudiantes se encuentran insatisfechos frente a la calidad de atención del respectivo Director de Escuela, Secretaría y Decano, así como la estructura y coherencia entre las asignaturas de la Malla Curricular.

En la Dimensión Equipo Docente se obtuvo una media de 3.05 , debido a que los estudiantes son indiferentes en cuanto a la calidad académica del profesorado, la puntualidad y cumplimiento de horas de clases, y la claridad en la exposición de contenidos.

En la Dimensión Infraestructura de la Facultad se obtuvo una media de 2.58 , debido a que los estudiantes se encuentran insatisfechos en cuanto a la disponibilidad del laboratorio de cómputo, los libros y material bibliográfico en la biblioteca y la calidad en los edificios de la Facultad.

Estos resultados indican que los estudiantes mantienen una posición de insatisfacción.

\section{Conclusiones}

La presente investigación determinó que la Calidad del Servicio influye significativamente en la Satisfacción de los Estudiantes de la Facultad de Ciencias Jurídicas y Empresariales. Las dimensiones de Calidad de Servicio: Elementos Tangibles, Confiabilidad, Capacidad de Respuesta, Seguridad y Empatía son percibidas como de calidad pésima; $y$ las dimensiones de Satisfacción del Estudiante, Métodos de Enseñanza y Aprendizaje, Organización de las Carreras e Infraestructura de la Facultad indican insatisfacción, mientras que las dimensiones Evaluaciones y Equipo Docente indican indiferencia por parte de los Estudiantes, además existe una correlación moderada de 0.596 entre ambas variables de estudio.

En relación a la variable "Calidad de Servicio", un 49.97\% Estudiantes de la Facultad de Ciencias Jurídicas y Empresariales perciben la Calidad de Servicio de manera negativa o pésima, debido a que los alumnos no califican de buena manera los servicios que se brindan en dicha facultad. Un $16.71 \%$ de los estudiantes lo percibe de manera positiva o excelente, y un $33.32 \%$ de los estudiantes se mantiene indiferente.

En relación a la variable "Satisfacción del Estudiante", un $30.47 \%$ de los Estudiantes de la Facultad de Ciencias Jurídicas y Empresariales se encuentran satisfechos con la Facultad, frente a un $33.15 \%$ que se encuentra insatisfecho, mientras que un $36.38 \%$ se mantiene indiferente.

\section{Referencias Bibliográficas}

- Wikipedia. (s.f.). Historia de la calidad. Disponible en http://es.wikipedia.org/wiki/Historia_ de_la_calidad

- Universia. (s.f.). Universia. Disponible en http://universidades.universia.edu.p e/gestion/calidad-universitaria/

- Rosander, A. (1994). Los catorce puntos de Deming aplicados a los servicios. Madrid, España: Editorial Díaz de Santos.

- Rodríguez, C, (s.f.). Investigación evaluativa en Educación, 2008-09. Obtenido de Calidad Educativa. Disponible en http://ocw.usal.es/ciencias-sociales1 /investigacion-evaluativa-eneducacion/contenidos/Calidad.pdf

- Castillo, S. (2002). Compromisos de la Evaluación Educativa (1a ed.). 
Madrid, España. En: Editorial Pearson Educación S.A.

- Vavra (2002). Evaluación de la Satisfacción de Clientes. Madrid, España: Editorial Ediciones Profesionales

Email: jerzylferez@hotmail.com

Recibido: 24/10/2014

Aceptado para publicación:

$12 / 12 / 2014$ 